\title{
EDUCACIÓN AMBIENTAL Y DISEÑO CURRICULAR
}

FERNÁNDEZ ROJERO, F. y MORENO RODRÍGUEZ, $M^{\text {a }} \mathrm{L}$. I.B. Mixto $n^{\circ}$ 3. Barrio Pesquero. Santander.

\section{SUMMARY}

After using and leaving an active methodology, authors describe a new curricular design for pupils of 1st of B.U.P. in the subject of Nature Science.

This new design has the following features: 1st. It tries to be based on the constructivist point of view of learning. 2on. It tries to include Environmental Education in the curriculum.

\section{INTRODUCCIÓN}

Durante los cursos 83-84 y 84-85, en el Seminario de Ciencias Naturales del X.B. del Barrio Pesquero de Santander, habiamos desarrollado una programación para $1^{\circ}$ de BUP con un enfoque ambiental, basada sobre todo en el uso de materiales (itinerarios, actividades, claves, etc...), que al tiempo ibamos diseñando en un programa de Educación Ambiental de un grupo de trabajo apoyado por el C.E.P. de Santander.

Entre nuestras hipótesis de partida se encontraban algunas que hacian referencia a que el planteamiento activo de enseñanza por descubrimiento guiado del entorno, aparte de ser necesario y factible, conseguiría:

1. Mejoras en el aprendizaje.

2. Mejora en los hábitos de trabajo, y

3. Mejora en la relación con el medio ambiente.

Como resultado de esta experiencia sólo se pudo comprobar como válida la $2^{3}$ de las hipótesis citadas; en las otras dos, resultó que no se obtenían mejoras ostensibles, a pesar de que los materiales fueron experimentados y rediseñados en numerosas ocasiones (Rojero 1987).

Una mirada a la bibliografia nos muestra que los modelos didácticos por descubrimiento, con una idea inductivista de la investigación y en ocasiones espontáneo como el que estábamos utilizando, se están revelando como poco consistentes (Hodson 1985, Gil 1986), al tiempo que se abre paso a un nuevo paradigma educativo, (al menos en la enseñanza de las Ciencias), basado en la investigación, tanto del alumno (Gil 1983,
Titone 1981, Tonucci 1986, Cañal 1987) como del pro* fesor (Stenhouse 1981, Elliot 1986, Gimeno 1983).

Las tendencias en la Educación Ambiental apuntan también en la línea del aprendizaje por investigación (Porlán y Cañal 1986, García 1987), dando a ésta una perșpectiva holistica y sistémica (Bertalanffy 1986, Jantsh 1986).

La necesidad de plantearse el curriculo de forma que sirviera no sólo a los objetivos de aprendizaje científico, sino también a los de actitudes, valores y respon. sabilidad, propios de la Educación Ambiental, expresados en la Carta de Belgrado, y necesarios en la sociedad democrática (Charles 1982), nos llevó a rediseñarlo $\mathrm{y}$ a introducir una metodología a la que hemos denominado «Método de Proyectos de Investigación", al estar basado en la metodologia de proyectos que consideramos adecuada para los objetivos más propios de ta Educación Ambiental, ya que consideramos como Legrand (1982) que: «El objeto de producción o de estudio y la actividad para realizarlo tienen un valor afectivo para el alumno; al cual se engancha voluntaria y personalmente, $y$ la intensidad de esta adhesión personal, caracteriza fundamentalmente el hecho de que haya o no proyecto. Como tal, el proyecto pertenece necesariamente a la pedagogía del aprendizaje, por oposición a la pedagogía de la enseñanza» y en el modelo de investigación (Cañal y Porlan 1987) que juzgamos necesarios para los objetivos de conocimiento enfocado todo ello desde una perspectiva constructivista del aprendizaje (Driver 1986). 
Nuestro nuevo modelo se encuentra por tanto en clara oposición, no sólo con nuestros planteamientos anteriores, sino con gran parte de los que se hacen en la actualidad en nuestro país, tanto en Educación Ambiental como en la Enseñanza de las Ciencias.

\section{METODOLOGIA}

El modelo se concreta en una sucesión cíclica de acciones pedagógicas (figura 1), encaminadas a conseguir que se produzca un proceso investigador del entorno.

Solemos comenzar la primera fase con una visita a una zona determinada, en la que se pretende casi exclusivamente despertar la curiosidad del alumno, al hacerle observar y descubrir cosas que normalmente le pa. san desapercibidas.

Para esta primera salida, se ha experimentado toda la gama que va desde la forma muy estructurada con una guía detallada de las observaciones y actividades a realizar (itinerarios de la Naturaleza) hasta aquélla poco o nada estructurada, en la que la observación se deja a la espontaneidad y a los intercambios verbales que el momento determine. La experiencia demuestra que esta $2^{\text {a }}$ forma débilmente estructurada es la preferida del alumno de $1^{\circ}$ de BUP, probablemente porque le da una mayor sensación de libertad y aventura (Bernáldez 1987).

De vuelta al centro, y con diferentes técnicas se propone a los alumnos que de forma individual primero, en grupo después y por último en gran grupo, expresen sus intereses y conocimientos tanto previos como actuales sobre el medio visitado, al considerar que en la intersección entre estos conocimientos e intereses, se encuentra el punto de partida para poder pasar de una observación simplista y anecdótica a una observación más reflexiva que le permita plantear interrogantes acerca de las cosas.

Es evidente que a pesar de ello, los alumnos, salvo excepciones se encuentran lejos de hacerse preguntas que tengan verdadero interés, planteando en su lugar cuestiones muy generales del tipo de:

figura 1

Sucesión de actividades a to largo del proceso.

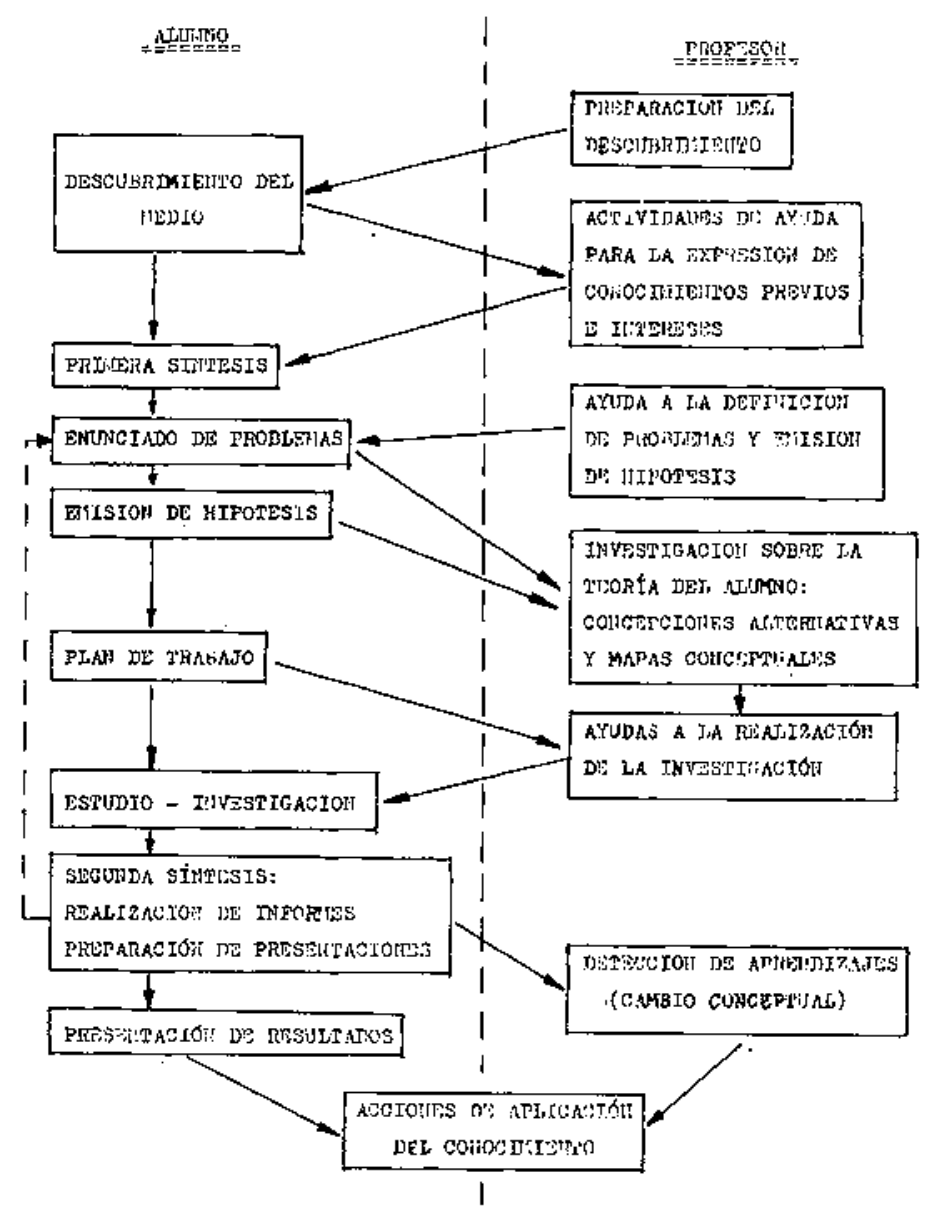


- ¿Cómo viven las aves?

- ¿Cómo se clasifican los árboles?, etc.

Es decir, son capaces de precisar el tema de su interés pero no de forma suficientemente concreta.

A partir de este punto, los alumnos forman grupos y deciden sobre cual de las preguntas planteadas quieren realizar su trabajo.

Comienza entonces un periodo de arduo trabajo para el profesor, en el que se pide a los grupos que imaginen posibles respuestas a su pregunta, varias mejor que una, razonando por qué podrian ser verdad. Lo cual lleva necesariamente a una concrecion mayor del problema, por ejemplo:

$-{ }_{i}$ Cuáles son las aves terrestres que viven en la Magdalena? o

- ¿Qué clases de palmeras viven en la Magdalena? (La Península de la Magdalena es un parque público que utilizamos con frecuencia para nuestro trabajo)

El paso siguiente es que se repartan las hipótesis enunciadas anteriormente, entre los miembros del grupo e intenten imaginar qué consecuencias en la naturaleza tendrian el que sus hipótesis fueran ciertas. St al efectuar esta operación surgen nuevas preguntas, se les pide que las anoten y repitan el proceso.

Cada cierto tiempo se hace una puesta en común, en gran grupo intentando que salgan a la luz las imprecisiones de su propuesta, por ejemplo:

- "Cómo el clima de aquí es más duro», o

- "Podrian vivir varias parejas de ratoneros?»

Durante esta fase de emisión de hipótesis, se les pasan algunos cuestionarios y se les proponen ejercicios de asociaciones de palabras, que junto con las propias emisiones de hipótesis permiten intentar conocer cuáles son sus concepciones sobre el problema, así como la estructura conceptual sobre la que se sustentan.

El profesor realiza su propio esquema conceptual sobre las propuestas emitidas, señalando qué conceptos son vislumbrados por el conjunto de los alumnos que trabaja sobre un tema y qué conceptos son los más próximos a éstos, así como el previsible camino que podria llevar a la construcción de un nuevo e importante concepto; los cuales el profesor considera así en función de los que fueron importantes en la Historia de la Ciencia y más concretamente de la Ecología (Giordan, 1987), y adjudicando al concepto de Ecosistema la característica de concepto estructurante (Gagliardi 1986).

Nosotros consideramos a título de hipotesis, que ha habido, a grandes rasgos, tres etapas en el desarrollo de las Ciencias Naturales:

$1^{\text {a }}$ - Una etapa de visión estática, en la cual se desarrollan dentro de la Biología, ramas como la Sistemática, la Morfología, la Anatomía, etc... $2^{a}$ - Una etapa de visión dinámica, en la que se desarrollan ciencias tales como la Embriología, la Genética, las Teorías Evolutivas, etc...

$3^{a}$.- Una etapa de visión compleja, en la que los problemas se abordan desde diferentes puntos de vista, o desde distintos niveles de organización, en la cual se desarrollan ciencias como la Ecología o la Biología Molecular.

Según nuestra experiencia los alumnos parecen reproducir tales etapas, por lo tanto no es de esperar que propongan problemas que superen a los de las fases 1 y 2 .

Al final de este proceso, y en algunos casos más adelante, durante la fase de estudio, los problemas han quedado concretados de forma suficiente; por ejemplo, dos grupos de alumnos plantearon:

- ¿Podrían vivir 607 parejas de ratoneros en el recinto de la Magdalena?

Hipotesis a investigar (elegida por etlos entre las consideradas): " $\mathrm{Si}$, porque existen gran variedad de aves de pequeño tamaño que podrían ser cazadas por ellos y proporcionar el alimento necesario».

- ¿Por qué sobreviven en la Magdalena la palmera y el palmito que son de otros climas más secos y calurosos?

Hipótesis a investigar: «Puede ser debido a las características similares del suelo".

Se les pide que diseñen un plan de estudio, en el que deben de señalar: la bibliografia a consultar, Ios trabajos de campo a realizar, y las personas, instituciones, etc... a las que se podría consultar, (no olvidemos que la propuesta se hace desde una perspectiva de fuerte implicación con el medio), reparto del trabajo y del tiempo, etc...

Recordemos en este sentido lo que dice Legran (1982): "La ejecución de un proyecto da lugar a una anticipación formal y colectiva de las fases de su desarrollo y del objetivo a conseguir».

La ejecución del proyecto suele llevar un tiempo largo, 3 o 4 semanas, en las que se alterna el trabajo de aula y laboratorio con las nuevas salidas al mismo medio.

En los casos en que la fase anterior tuvo éxito, es fácil; el alumno sabe lo que tiene que buscar y actúa en consecuencia. En otros casos, se pierde en un activismo bastante infructuoso que, aunque intentamos reconducir, en general respetamos.

Los dos grupos a los que estamos haciendo referencia en el trabajo, consultaron mucha bibliografia: claves de aves, enciciopedias de la naturaleza (que curiosamente un gran numero de alumnos tienen en sus casas), trabajos de otros alumnos, libros de texto, libros sobre prácticas de laboratorio, etc... 
Como consecuencia de ello, el primer grupo averiguó que los ratoneros son territoriales, Io que les llevó inmediatamente a considerar que el problema ya estaba resuelto, la intervención del profesor les hizo ver la conveniencia de seguir investigando acerca del alimento, ya que era mejor tener más pruebas.

Estos alumnos habian desarrollado una cierta habilidad para reconocer las aves, $y$ argumentaban siempre que habia muchas; el profesor les instó a que calcularan si realmente permitirían la alimentación de los ratoneros como ellos suponían. A raíz de ello se dedicaron a batir zonas de terreno en las que contaban el número de aves de cada especie que veían. El cálculo de la biomasa de las pequeñas aves les permitió comprobar que en una estimación aproximada ésta era suficiente; aunque estaban convencidos de que el problema ya estaba resuelto. Los conceptos de nivel trófico $y$ de ajuste entre los niveles tróficos (Economía de la Naturaleza, Giordan 1987) quedaron a punto para construirse...

El otro grupo, a partir de un libro que encontraron en el laboratorio, se dedicaron a analizar los suelos en los que crecian la palmera y el pamito, para ver si tenian las mismas características que la bibliografía adjudicaba a los de los climas desértico y mediterráneo. La composición fuertemente arenosa y caliza, les convenció de que su hipotesis era correcta y se dieron por satísfechos. Importantes conceptos como el de limitación de la vida por los factores abióticos y la consiguiente distribución geográfica de las especies aparecieron en su horizonte.

Cuando terminan sus investigaciones, preparan sus informes y presentaciones al gran grupo, producciones que el profesor les «compra» en forma de calificaciones, para lo cual rellenan una parrilla de puntuación que los alumnos conocen. Tras la sesión de presentación se establece un debate entre los demás alumnos y el grupo.

El grupo de aves realizó una presentación clásica, expositiva, ayudándose de dibujos y de un mapa. El de suelos, simuló un programa de Televisión en el que los miembros cran los corresponsales en diferentes lugares (La Magdalena, el desierto, zona de clima mediterráneo).

Es de notar que en esta fase de presentación, los diferentes grupos han utilizado ya practicamente toda la gama de posibilidades, y que en la fase de estudio se han relacionado con todo tipo de instituciones y personas (Icona, Ayuntamiento, Consejería de Medio Ambiente, Museo Oceanográfico, Ecologistas, etc...); en los dos cursos que llevamos utilizando la metodología descrita.

\section{ORGANIZACION Y RECURSOS}

Creemos al igual que Ausubel (1978) que el pequeño grupo es la única forma factible de organización de la clase. Estos grupos se forman siempre en función de los intereses de los alumnos; asumiendo que estos intereses son complejos, al intervenir en ellos factores afectivos y sociales, externos a la dinámica de la clase, y que la resolución del conflicto entre ellos resulta formativa para el alumno.

En cuanto a material bibliográfico, el no utilizar un li* bro de texto determinado, así como el haber ido configurando una buena biblioteca en el seminario permiten una gran diversidad de fuentes.

Las salidas de campo se realizan siempre los martes por la tarde, para lo cual los horarios de profesores y alum. nos están «arreglados» desde principio de curso.

\section{EVALUACrón}

Diferenciaremos para empezar entre evaluación forma. tiva y sumativa; como ya hemos indicado, no hay exámenes y las calificaciones se obtienen a partir de los trabajos realizados.

Nos referiremos ahora a los aspectos formativos de la evaluación, entendiendo ésta como un proceso investígador de lo que ocurre en el aula (Stenhouse 1984, Elliot 1986).

Esta evaluación, investigativa y cualitativa, intenta sobre todo solucionar problemas, siguiendo para ello un esquema adaptado de otro propuesto por Elliot (1986).

Son muchos los problemas que se plantean al utilizar un modelo tan novedoso para nosotros como el presentado, y obviamente el tiempo disponible no permite investigarlos todos.

Nuestro trabajo se centra sobre todo en tres problemas:

- Racionalización y validación del modelo.

- Estructura conceptual del alumno, teorias alterna. tivas y su evolución: cambio conceptual.

- Evolución de las actítudes ante el propio trabajo y ante el medio ambiente, y su relación con cl aprendi. zaje conceptual significativo.

Las técricas e instrumentos utilizados son:

- Diarios de clases de profesores y de alumnos.

- Análisis de informes y producciones escritas.

- Cuestionarios, ejercicios, tests.

- Análisis de las discusiones de los grupos.

- Observación sistemática.

Tras el tiempo que llevamos de experimentación podemos ir adelantando algunos resultados:

$1^{\circ}$. - Los alumnos tienen serias dificultades para emitir hipótesis o al menos para hacerlas explícitas. No po. seemos datos suficientes pero las investigaciones reali. zadas indican que los alumnos no poseen un pensamien. to formal suficientemente desarrollado, o bien no poseen la suficiente estructura conceptual de la materia que les permita tener en qué asentarse. 
La tendencia a plantear problemas alejados de sus conocimientos, influye también en esta dificultad.

Cuando se detectan dificultades como las citadas, el profesor debe permitir un planteamiento más próximo a la búsqueda (Gil 1986) que a la investigación, al no requerir un nivel tan elevado de formalización.

$2^{\mathbf{a}}$. - Tendencia al activismo, los alumnos tienden a trabajar sobre el tema del problema, pero no en su resolución.

Creemos que su experiencia escolar previa, también en otras asignaturas, así como su "motivación aversiva» (Novak 1982), es decir miedo a una mala calificación escolar, son las responsables de este problema; también algunas actitudes involuntarias del profesor (currículo oculto). Pero al valorar el esfuerzo, aunque no esté bien encaminado, hace que el alumno se reafirme en este planteamiento, lo que por otra parte le da una «tranquilidad» ante el riesgo académico (Doyle 1977).

$3^{a}$. - Se aprecia una gran tendencia a la falta de rigor en la toma de datos, admitiendo errores bastante grandes (Metodología de la superficialidad, Carrascosa y Gil 1985), to que nos hace pensar en el nulo contacto con la metodología experimental por parte de nuestros alumnos, con lo cual la necesidad de medir todavía no ha surgido en ellos y, si lo ha hecho, ha sido sin la necesidad de que las modidas sean exactas. (Al preguntarles por su experiencia previa en el uso de instrumental de laboratorio, más del $90 \%$ no habian nunca utilizado microscopios, balanzas, etc...).

$4^{\mathrm{a}}$. - Idea equivocada sobre el trabajo científico. $\mathrm{Pa}$ ra ellos la manipulación del objeto no encaminada a comprobar una hipótesis, y sin ningún tipo de control de variable, equivale a una experimentación.

Por otra parte, tienen tendencia a repetir prácticas que encuentran en los libros, sin saber exactamente para qué lo hacen.

$5^{2}$.- Falta de espíritu crítico; para ellos lo que viene en los libros, o lo que dicen personas mayores equivale a una comprobación de sus hipótesis. Al tiempo que tienen la idea de que realizar una investigación, no es encontrar respuestas a preguntas, sino acumular datos sin ningún criterio. Creemos que en cierto modo esta-

\section{REFERENCIAS BIBLIOGRÁFICAS}

AUSUBEL, D.P., 1978. Psicología Educativa: un punto de vista cognoscitivo. (Trillas: México).

BERNÁLDEZ, F.G., BEYANA, J. y LUCIO, J.V., 1987. Percepción Ambiental y Educación Ambiental, el rol de los centros de interés, Educación Ambiental, 1, pp. 25-30.

BERTALANFFY, L., 1968. Teoría General de Sistemas. (Fondo de Cultura Económica: Madrid). mos trabajando contra su experiencia académica pre* via y actual.

Con la misma prudencia que hemos utilizado en los aspectos más conflictivos, nos referimos ahora a los aspectos positivos más relevantes que hemos encontrado.

$1^{\circ}$.- El aprendizaje significativo de conceptos (Ausu* bel 1978), se va produciendo poco a poco, rompiendo con la inercia memorística, que ha llevado a que los alumnos cuando llegan a nosotros, sean incapaces de hablar de ningún tema científico, ni establecer las minimas relaciones entre conceptos. Para poder afirmar más acerca de este aspecto, tendremos que esperar un tiempo para hacer pruebas de permanencia de conceptos.

$2,^{\circ}-$ Desde la perspectiva constructivista en la que nos hallamos, el aprendizaje de conceptos no puede separarse de los aspectos de procedimiento y actitud. Nuestros datos apuntan que una vez superado el "susto» inicial ante nuestros métodos, el alumno empieza a estar motivado además de por los aspectos académicos, por otros tales como el impulso cognitivo y el mejoramiento del yo en términos de Novak (1972), con el consiguiente cambio en sus formas de actuar en el aula.

$3^{\circ}$. - La mejora en el «clima» de la clase es evidente; la libertad, la cooperación, el respeto mutuo, son valores de los que no se hablan, sino que se usan.

El trabajo en equipos, cuando el alumno es de verdad responsable de su propio aprendizaje, permite que las diferencias personales en cuanto a capacidades y conocimientos previos, no sólo no perturben el proceso didáctico, como ocurre en otras metodologías, sino que por el contrario constituyan una fuente de riqueza en los intercambios personales y en el trabajo en equipo. Aqui resulta que lo valioso no es la uniformidad, sino la diversidad en las personas.

$4 .^{\circ}$ - No tenemos todavía datos acerca de un cambio medible en las actitudes hacia el Medio Ambiente, aunque entre nuestras hipótesis de partida está la de que este cambio sólo es posible cuando se construye un buen conocimiento cientifico acerca de la dinámica medioambiental, (salvo que se persiga un simple adoctrinamiento), y en este proceso es en el que nos encontramos.
CAN̄AL, P., 1987. Un enfoque curricular basado en la investigación, Investigación en la Escuela, Vol. 1, pp. 41-50.

CAN̄AL, P. y PORLAN, R., 1987. Investigando la realidad próxima: un modelo didáctico alternativo, Enseñanza de las Ciencias, Vol. 5, pp. 136-145.

CARRASCOSA, J. y GIL, D., 1985. La «metodología de la superficialidad» y el aprendizaje de las ciencias, Ense- 
ñanza de las Ciencias, Vol. 3 (2), pp. 113-120.

CHARLES, M., 1982. L'environnement, espace educatif. (Arcidep: Paris).

DOYLE, W., 1977. Learning the Classroom Environment: An Ecological analysis, Journal of Teacher Education, Vol, 28 (6), pp. 51-55.

DRIVER, R., 1986. Psicología cognoscitiva y esquemas conceptuales de los alumnos, Enseñanza de las Ciencias, Vol. 4, pp. 49-53.

GAGLIARDI, R., 1986. Los conceptos estructurantes en el aprendizaje por investigación, Enseñanza de las Ciencias, Vol. 4 , pp. $30-36$.

GARCIA, J.E., 1987. La interacción con el medio en relación con la investigación en la Escuela, Investigación en la Escuela, Vol. 1, pp. 57-62.

GIL, D., 1983. Tres paradigmas básicos en la Enseñanza de las Cicncias, Enseñanza de las Ciencias, Vol. 1, pp. 26-34.

GIL, D., 1986. La metodología cientifica y la Enseñanza de las Ciencias: unas relaciones controvertidas, Enseñanza de las Ciencias, Vol. 4, pp. 111-122.

GIMENO, J., 1983. EI profesor como investigador en el aula: un paradigma en la formación de profesores, Educación y Sociedad, 2, pp. 51-75.
GIORDAN, A., 1978. La Enseñanza de las Ciencias. (Pablo del Río: Madrid, 1982).

GIORDAN, A., 1987. Histoire de la Biologie. (Lavoisier: Paris),

HODSON, D., 1985. Philosophie of Science, Science and Science education, Studies in Science Education, 12, pp. 25-27.

JANTSH, E., 1980. Interdiciplinariedad: sueños y realidad, Perspectivas, Vol. 10 (3), UNESCO. París.

LEGRAND, L., 1982. Pour collège démocratique. (La documentation Francaise: París).

NOVACK, J.D., 1978. Teoria y Práctica de la Educación. (Alianza Universidad: Madrid).

PORLAN, R. y CAÑAL, P., 1986. Más allá de la investigación del medio, Cuadernos de Pedagogia, 142, pp. 8-12.

ROJERO, F.F., 1987. I,iencres: del aula de la naturaleza a la naturaleza del aula, Quima, 14, pp. 43-44.

STENHOUSE, L., 1984. Investigación y desarsollo del curriculo. (Morata: Madrid).

TITONE, R, 1981. Psicodidóctica. (Narcea: Madrid).

TONUCCI, F., 1976. La escuela como investigación. (Avance: Barcelona). 